\title{
Impact of a new diagnoses thesaurus on the French ED syndromic surveillance system
}

\author{
Cécile Forgeot $^{1}$, Isabelle PONTAIS ${ }^{1}$, Emmanuel Dos Ramos ${ }^{4}$, Gilles Viudes ${ }^{3}$, Christophe \\ Vincent-Cassy ${ }^{2}$, François Dubos ${ }^{3}$, Anne Fouillet ${ }^{1}$, Céline Caserio- Schönemann ${ }^{1}$ \\ ${ }^{1}$ DATA, Sante publique France, Saint-maurice, France, ${ }^{2}$ SFMU, French society for emergency healthcare, Paris, France, ${ }^{3}$ Fedoru, Emergency \\ regional observatory Federation, Paris, France, ${ }^{4}$ ORUPACA, PACA Emergency Network Observatory, Hyères, France
}

\section{Objective}

The study aims to evaluate the potential impact of the revision of the thesaurus used by ED physicians to code medical diagnoses, on the syndromic indicators used daily to achieve the detection objective of the French syndromic surveillance system.

\section{Introduction}

As part of the French syndromic surveillance system SurSaUD®, the French Public Health Agency (Santé publique France) collects daily data from the emergency department (ED) network OSCOUR ${ }^{\circledR}$ [1]. The system aims to timely identify, follow and assess the health impact of unusual or seasonal events on emergency medical activity.

Individual ED data contain demographic (age, gender, residence zip code), administrative (dates of attendances and discharge, ED, etc.) and medical information (chief complaint, main and associated medical diagnoses, severity). Medical diagnoses are encoded using the ICD10 classification. Then syndromic groups are built based on these ICD10 codes for ensuring syndromic surveillance in routine.

Even if ICD10 is recommended on the national guidelines for coding ED attendances, this thesaurus offers a too large variety of codes. Particularly, it includes lots of diseases that may never be observed or confirmed in ED. This variety let selection of the appropriate codes difficult for physicians in a reactive use and could discourage them to code diagnoses.

In order to encourage appropriate and reactive coding practice, we decided in 2017 to produce a new diagnoses thesaurus with a limited list of ICD10 codes. Then a committee of medical and epidemiological experts was created by the Federation of regional emergency observatories (FedORU), to propose an operational thesaurus that includes relevant codes for both ED in a daily routine practice and syndromic surveillance.

\section{Methods}

The committee has met 10 times since 2017. Since it would have been hard to work on the complete ICD10 list, the work was based on a more limited thesaurus already used by part of French ED. Only codes, which were pertinent regarding ED activity and interest for public health alert, have been considered. The main principles that have guided the selection were to 1) keep codes related to diagnoses that physicians are able to diagnose on a clinical basis or with rapid diagnostic tests, 2) remove diagnoses providing redundant information regarding other variables (such as circumstantial information) and 3 ) ensure that a substitution code was kept when a removed code was frequently used or was of interest for syndromic surveillance.

Among the 86 syndromic groups defined on the basis of a list of ICD10 codes selected in the complete thesaurus, 34 are daily analyzed by Santé publique France for outbreak detection and early assessment of public health events. Those 34 syndromic groups have been recalculated by considering the revised thesaurus on a three-year period (from 2015 to 2017) at national level.

In order to measure the potential impact of the revised thesaurus on the syndromic groups, we have considered three evaluation measures: 
1. the proportion of ICD10 codes deleted (removal rate) from the initial definition of each syndromic group, due to the limitation of the thesaurus (calculated for the 86 syndromic groups);

2. the mean difference in the daily number of attendances between the initial and the new versions of each syndromic group (calculated for the 34 syndromic groups);

3. the linear correlation coefficient between the daily numbers of attendances of the initial and the new version of each syndromic group, in order to assess if the daily fluctuations of the new syndromic group are similar to those of the initial syndromic group (calculated for the 34 syndromic groups).

\section{Results}

Among the 86 syndromic groups, 75 (85\%) have been impacted by the revised thesaurus, which implied codes removal. Among those 75 syndromic groups, the number of ICD10 codes included in their definition has been reduced by $71 \%$ on average. This removal rate varied between $17 \%$ and $100 \%$. Syndromic groups including initially more than 100 codes have been the most concerned by a limitation of the number of ICD10 codes.

Among the 34 syndromic groups daily analyzed for outbreak detection, 32 have been impacted by code removal with a mean removal rate of $68 \%(0 \%-97 \%)$. On average, $77 \%$ of daily attendances have been retained by the new version of syndromic groups, varying from $15 \%$ to $100 \%$. Only 3 syndromic groups have kept less than $60 \%$ of attendances: Decrease of well-being (36\%), Conjunctivitis (32\%) and Hypothermia (15\%).

On average, the correlation coefficient has been of 0.96 , varying from 0.57 to 1 . The lowest values have been observed for the same three syndromic groups listed above: Decrease of well-being (0.57), Conjunctivitis (0.91) and Hypothermia (0.59). 18 among the 34 syndromic groups had a correlation coefficient higher than 0.99 .

\section{Conclusions}

The study showed that most of the syndromic groups were impacted by the revised thesaurus, which resulted in a removal of about two thirds of the ICD10 codes usually considered in daily surveillance. However, more than three quarters of attendances were still retained in the new syndromic groups. This new thesaurus was conceived to rationalize the number of diagnoses codes but a substitution code was systematically proposed to replace removed codes.

Those results highlighted that a large number of codes included in the complete ICD10 thesaurus were rarely used and that the most frequent codes were kept in the revised thesaurus version. However, this study showed that a few syndromic groups were strongly impacted by the revised thesaurus and can suffer of reduced performances to detect unusual variations. Based on those results, a second round of exploration of specific parts of the complete ICD10 thesaurus will be necessary to adapt either syndromic groups or the revised thesaurus. Even if the number of attendances may be reduced due to the removal of ICD10 codes, temporal variations remain similar for the majority of syndromic groups. Syndromic surveillance system does not aim to provide exhaustive quantification of attendances for a pathology, but aims to be able to detect expected or unusual public health variations. These evaluation results correspond to the worst-case scenario assuming that ED physicians will not modify their encoding habits by using the substitution codes but keep using their current thesaurus. However, we expect that this new and simplified version will facilitate diagnosis encoding task and lead toward a better diagnosis encoding rate. Once this new thesaurus will be widely used, we can expect a substantial improvement of the quality of ED medical data and then of syndromic surveillance results.

Finally, this study enhances the importance that both data providers and epidemiologists in charge of syndromic surveillance work closely, in order to improve system in shared objectives.

\section{References}

1. Fouillet A, Bousquet V, Pontais I, Gallay A, Caserio-Schönemann C. 2015. The French emergency department OSCOUR network: evaluation after a 10-year existence. Online J Public Health Inform. 7(1), e74.

https://doi.org/10.5210/ojphi.v7i1.5740 